\title{
Review
}

\section{Management of Parkinson's Disease 20 Years from Now: Towards Digital Health Pathways}

\author{
Jochen Klucken $^{\mathrm{a}, \mathrm{b}, *}$, Rejko Krüger ${ }^{\mathrm{c}, \mathrm{d}}$, Peter Schmidt ${ }^{\mathrm{e}}$ and Bastiaan R. Bloem ${ }^{\mathrm{f}}$ \\ ${ }^{a}$ Department of Molecular Neurology, University Hospital Erlangen, Friedrich-Alexander University (FAU) \\ Erlangen-Nürnberg, Germany \\ ${ }^{\mathrm{b}}$ Research Group Digital Health Pathways, Fraunhofer Institute for Integrated Circuits (IIS), Erlangen, Germany \\ ${ }^{\mathrm{c}}$ Luxembourg Centre for Systems Biomedicine (LCSB), University of Luxembourg, Luxembourg \\ ${ }^{\mathrm{d}}$ Centre Hospitalier de Luxembourg (CHL), Luxembourg \\ ${ }^{\mathrm{e}}$ Brody School of Medicine, East Carolina University, Greenville, NC, USA \\ ${ }^{\mathrm{f}}$ Department of Neurology, Radboud University Medical Center, Donders Institute for Brain, Cognition and \\ Behavior, Nijmegen, The Netherlands
}

Accepted 29 November 2018

\begin{abstract}
Current best medical treatment for patients with Parkinson's disease (PD) involves a medical professional who applies state-of-the-art knowledge of diagnostics and treatment-as derived from cohort studies and clinical trials-to the healthcare process of individual patients. Thus, the much-needed personalization of medicine depends on the abilities, experience and intuition of medical professionals to adjust group-based knowledge to individual decision making. Within 20 years from now, such personal clinical decisions will be largely supported by digital means, also defining a new ecosystem of healthcare often referred to as "digital medicine". We expect that the next phase of digitalization will include new "digital health pathways": data-driven personalized decision support that is based on a combination of multimodal data sources, including evidence-based medical knowledge (e.g., clinical guidelines), personal disease profiles (including genetic determinants of disease progression and treatment response), insights into individual disease trajectories (thereby defining subgroups of patients) and individual patients' needs. Here, we illustrate the potential of this development by sketching the contours of a digitally supported care pathway for gait disability and falls. Such digital health pathways will support the introduction of personalized medicine for PD patients, allowing patients to benefit optimally from individually tailored treatments. This should result in a better quality of life for patients and lower costs for society.
\end{abstract}

Keywords: Parkinson's disease, management, treatment, digital pathways, innovation, healthcare

\footnotetext{
*Correspondence to: Jochen Klucken, MD, University Hospital Erlangen, Kopfklinikum, Schwabachanlage 6, 91054 Erlangen, Germany. Tel.: +49 913185 39324; E-mail: jochen.klucken@ukerlangen.de.
}

\section{CHALLENGES IN THE CURRENT MANAGEMENT OF PARKINSON'S DISEASE}

Today's medicine is largely separated into two worlds. On the one hand, there is evidence-based medicine that is grounded on knowledge derived from carefully controlled studies on very distinct-and 
highly stratified-patient cohorts. Recruitment of patients is based on well-defined and typically strict criteria for inclusion and exclusion, and the calculation of treatment efficacy is based on averaged group results. On the other hand, there is real-life care which involves a one-to-one treatment context defined by the complexity of the patient's individual symptoms and comorbidities, and that relies upon the expertise and personal experience of healthcare professionals. Both worlds are currently parallel realities, and integration of both is needed. Indeed, randomized controlled studies (RCTs) are crucially important to increase our understanding of treatment effects and disease mechanisms, but there is insufficient RCT evidence to inform care guideline development. Only $11.5 \%$ of guideline recommendations were supported by level of evidence A support [1]. Furthermore, the available medical evidence cannot be translated easily into everyday clinical care practice, where patients are seen that may differ markedly from the carefully selected (and often highly motivated) individuals that participated in clinical trials. For example, real-life patients can be different because they are too old to be included in trials, or because they have co-morbid conditions potentially interfering with the pure intervention effect and hence be precluded from classical clinical trials. A good illustration here is pharma research, where clinical trials typically provide some kind of proof how new drugs can generally help (a group of) patients, but no insight into the treatment effect at an individual level and often no insight into comparative efficacy versus existing options.

The future of medicine will be largely defined by digitalization of healthcare procedures including new approaches to diagnostics, therapy, and communication between patients and their healthcare provider team. Innovative patient-centered healthcare technologies provide better medical information (outcome/target parameters), while medical data management platforms can make the medical information available to all stakeholders, ultimately supporting a new ecosystem of healthcare: "digital medicine". New research approaches towards digital medicine focus on analyzing the informational content of complex and large medical data arrays from multiple different data sources, including public domain (medical claims, wearable sensors, etc.) and individual data (genomics, metabolomics, transcriptomics, etc.). This approach is also referred to as big-data research that should allow for more precise, objective and comprehensive diagnostic measures and therapeutic options.
"Precision medicine", in common parlance restricted to genomic medicine, is better defined in this domain. As a whole person approach, it aims to leverage information/data for each individual patient. Current research in this field contributes to a better understanding of the relation between multimodal data and different groups of patients. Such knowledge will eventually support our ability to optimally tailor treatments based on the individual patients' profile. A major challenge now is to translate this understanding of precision medicine into individualized patient care, with a big focus on "tailoring" information from big data to personal applications. However, to transform our understanding of new technologies and medical data acquired in large cohort studies to individual applications requires a substantial paradigm shift from physician-centered "clinical guidelines" to patient-centered "clinical pathways". This development also requires a detailed insight into each person's disease course over time, and how this course is affected by medical management or lifestyle factors. These insights will allow us to standardize medical information for new digital benchmarking concepts, with development of a new digitalization process that supports the introduction of patient-centered medical workflows, with involvement of interdisciplinary and comprehensive healthcare services as well as IT-based and datadriven patient-management concepts.

Parkinson's disease (PD) is an ideal model disease to illustrate both the tremendous potential but also the limits of these new developments, for various reasons: (a) a wide range and complex interplay of motor as well as non-motor symptoms and treatment benefit and risk tradeoffs limit the quality of life for the individual patient; (b) the disease course differs markedly from patient to patient, and so does the response to the available treatments; and (c) the current treatment decisions are still almost exclusively based on history taking and clinical examination during rare point-ofcare visits, which offer insufficient insights into the patient's actual functioning in their own home environment [2]. Today, the much-needed personalization of medicine for PD patients still depends largely on the abilities, experience and intuition of treating physicians, nurses and allied healthcare professionals to adjust evidence-based medicine to individual decision making. Here, we will illustrate how new technological development, and more specifically the introduction of a personalized clinical pathways "the digital health pathway" - can support both professionals and patients in reaching medical decisions 
that are optimally tailored to their individual needs and profiles.

\section{HEALTHCARE GUIDELINES AND STANDARDS}

There are various routes to improve the quality of care (Table 1). Current healthcare delivery is based largely on clinical practice guidelines that are being developed by PD expert associations in various regions in the world. These guidelines are typically based on a combination of evidence-based medicine, with knowledge derived from clinical trials plus practice-based evidence (the professional expertise). Examples include the guidelines of the National Institute for Health and Care Excellence (NICE) in the UK [3] that aim to support clinical decisions of healthcare professionals and understanding and participation of patients and caregivers [4]. In Germany, the Association of the Scientific Medical Societies together with the German Society for Neurology have recently updated the S3 guidelines for PD [5] aiming to integrate evidence criteria for clinical decisions, diagnostics, and disease management primarily from the viewpoint of doctors and related healthcare professionals. For physiotherapists, the European Physiotherapy Guideline for PD was developed to harmonize and improve PD care with a strong focus on the viewpoint of physiotherapists [6]. In Canada, PD guidelines for pharmacists have been developed [7], and the American Academy of Neurology constantly updates different treatment aspects of PD in their guidelines on the treatment of non-motor symptoms of Parkinson disease [8]. Importantly, these guidelines typically offer generic support for healthcare professionals by clarifying which treatments are generally effective and should therefore be considered, but offer little guidance on how to apply this knowledge to the personal profiles, perspectives and needs of single patients.

Improved technologies and digitalization strategies in medicine are currently changing the way we conduct healthcare, with three major driving factors. First, mobile healthcare technologies including wearable sensors and smartphone-applications are becoming available that have the potential of providing objective, longitudinal and fine-grained information about the functioning of individual patients in their own home environment. Second, electronic health records and digital patient management platforms can now connect the different healthcare professionals, technologies and individual patients in an unprecedented manner. And third, the introduction of "big data" research-analysis of large and comprehensive datasets, derived from large and unselected populations - can potentially improve our knowledge on disease mechanisms, diagnostic and therapeutic strategies, not only at the overall group level but also at the level of smaller targeted groups, and possible even at the level of individual patients. Currently, prognosis building and medical

Table 1

Types of translational research and strategies to improve healthcare

\begin{tabular}{|c|c|c|}
\hline $\begin{array}{l}\text { Strategies to improve } \\
\text { healthcare }\end{array}$ & Characteristics & Goal of the strategy \\
\hline $\begin{array}{l}\text { A) Randomized } \\
\text { controlled trials }\end{array}$ & $\begin{array}{l}\text { Stratified cohorts of well-defined } \\
\text { patients, tested under carefully } \\
\text { controlled experiments }\end{array}$ & $\begin{array}{l}\text { Provide best evidence for efficacy of } \\
\text { selected treatments, based on averaged } \\
\text { group results }\end{array}$ \\
\hline $\begin{array}{l}\text { B) Clinical Practice } \\
\text { Guidelines }\end{array}$ & $\begin{array}{l}\text { Guidelines for healthcare } \\
\text { professionals }\end{array}$ & $\begin{array}{l}\text { Translate best evidence and combining } \\
\text { this with practice-based evidence } \\
\text { (professional experience) to arrive at } \\
\text { best care recommendations }\end{array}$ \\
\hline C) Training models & $\begin{array}{l}\text { Training modules for healthcare } \\
\text { professionals } \\
\text { Improving patient participation } \\
\text { Improving communication }\end{array}$ & $\begin{array}{l}\text { Implement guidelines into integrated } \\
\text { care models }\end{array}$ \\
\hline D) Big Data research & $\begin{array}{l}\text { Large and comprehensive data } \\
\text { sets from patient cohorts }\end{array}$ & $\begin{array}{l}\text { Data-driven analyses, aiming to better } \\
\text { understand the relation between } \\
\text { pre-defined parameters and treatment } \\
\text { outcomes or prediction in individual } \\
\text { patients } \\
\text { Exploratory analyses to identify novel } \\
\text { parameters that correlate to treatment } \\
\text { outcomes or improved patient } \\
\text { stratification }\end{array}$ \\
\hline
\end{tabular}


decision making are based on fairly crude measures such as presence of tremor (which may signal a more favorable prognosis) or late age at onset (which is more likely associated with early development of falls and cognitive decline). But ideally, professionals and patients would like to have insights into the personal clinical path that lies ahead, including information as to how this trajectory can be modified by specific management advice, also encompassing lifestyle changes. This personalized medicine approach, where the individual patient really moves into the center of healthcare strategies, should be able to provide individually tailored and objective real-life outcomes and benchmarks, thereby increasing transparency and supporting patients to contribute and participate in decision making [9].

Digitalization in healthcare has certainly reached the field of PD [10, 11]. Mobile healthcare technologies such as wearable sensors and smartphone applications aim to measure targeted parameters in a more accurate and objective way [12]. They will generate relevant medical information from the real-life home environment, and as such better reflect the disease-related burden and impairment of everyday life activities [13]. There is a separate chapter in this special issue dealing with these important developments [14]. Here, we focus on another important element of digitalization that includes new "digital health pathways": data-driven personalized decision support that is based on a combination of multiple big data sources, including the knowledge derived from mobile healthcare technologies. We will illustrate the potential of this development by sketching the contours of a digital care pathway for gait disability and falls.

\section{DIGITAL HEALTH PATHWAY CONCEPT}

Mobile healthcare technologies require a novel concept in order to provide clinical decision support. It is not sufficient that they merely provide more accurate outcome parameters or more fine-grained information. In order to support the individual patient's needs, the novel parameter or information provided by innovative healthcare technologies must be integrated into the complex healthcare workflow. One example is Kaiser Permanente's eCare for Moods (whose technology was developed by one of us [PS]) that provided a web-based interface for home-based weekly self-monitoring of depression and bipolar disorder, with updates and secure communications with the care team, which was demonstrated to be more effective than traditional outpatient care alone in an RCT [15]. Remote- and self-management technologies typically detect broad signals combining inputs from the environment, individuals, and their pathologies. These various signals must be combined with other data to optimize sensitivity and specificity of the information offered to support clinical decisions. Thus, clinical expertise and guidelines have to be mapped to the sensor signal domain to optimize use of wearable devices, both in informing self-management and in closing the loop of clinician guidance. Such optimization includes two complementary approaches: evaluation of sensor data while the patient phenotype is directly observed by an expert clinician; and evaluation of routine usability of the technology while individuals are engaged in their usual routine. Both approaches are needed, because new parameters that have been validated in standardized test paradigms may not translate automatically into ambulatory monitoring test scenarios. Even standardized tasks, such as finger taps or spiral drawing, may yield different results because of environmental effects without change in patient status. Work is still needed to establish robust parameters for PD signs and symptoms that are invariant across environments where the test is performed, and that are not affected by variations in test execution, with or without supervision, and with minimal (or quantified) training effects. New reporting concepts must also be defined: based on the sensitivity and specificity of the test and the severity of the indicated phenotype, should results be reported to the patient or a specific member of the care team? In Kaiser Permanente's eCare program, all alerts were initially shown to a research nurse but subsequent iterations limited care team alerts to those determined to be clinically significant. Such usability issues have challenged designers of user interfaces of electronic medical records: how to visualize parameters in order to support clinical decisions or patient participation using either normative values (from cohorts), or individualized time-series results? The resulting workflow is similar to clinical pathways that are used to structure multidisciplinary care for specific diseases and specific clinical targets. These classical clinical pathways are defined from the healthcare provider's perspective and translate clinical guidelines into local protocols and clinical practice $[16,17]$. However, clinical pathways relevant for the application of digital technologies would have to be defined from the patient's perspective. Thus, in order to enable the application of new mobile healthcare 
technologies and the digital management of outcome parameters and related information, we introduce the new concept of Digital Health Pathways (DHPs).

A DHP can be defined as a patient-centered medical workflow for the individual healthcare technology application. With a strong focus on the application of mobile healthcare technologies that (tele-)monitor the treatment effects within an individual patient, a DHP is fully patient-centered (in contrast to classical clinical guidelines that aim to support the clinical decisions of medical professionals). As such, a DHP guides the application of technology and thereby defines the integration of medical data into the healthcare process. For mobile healthcare technology devices, the DHP also provides a basic form of a user manual that standardizes how the respective device is being applied within the workflow of the patient and its healthcare team. DHPs are optimized around medical data relevant for each treatment process and how the data are recorded, analyzed, and communicated to the stakeholder for the distinct workflow.

\section{A DIGITAL HEALTH PATHWAY FOR GAIT AND FALLS}

Gait and falls are relevant treatment targets in PD that limit patients' mobility, independency and quality of life. In our EIT-Health project "MoveIT" we combine an inertial sensor-based gait analysis system $[18,19]$ with sensor-based fall detection [20] in a comprehensive assessment battery for clinical trials and care [21]. The application of the gait sensor system, or the fall detection by the pendant worn around the neck, is guided by the needs of the patient and defined by the DHP for gait and falls. As such, the DHP for gait and falls is a patient centered guideline that not only defines the application of the different aspects of technology-based assessment, but at the same time, much stronger includes the patient as a part of the healthcare team. Here, we will illustrate the concept of DHPs for the management of gait and falls (Fig. 1), but the concept is obviously much more widely applicable. Any DHP for long-term care has several generic steps in the pipeline of the
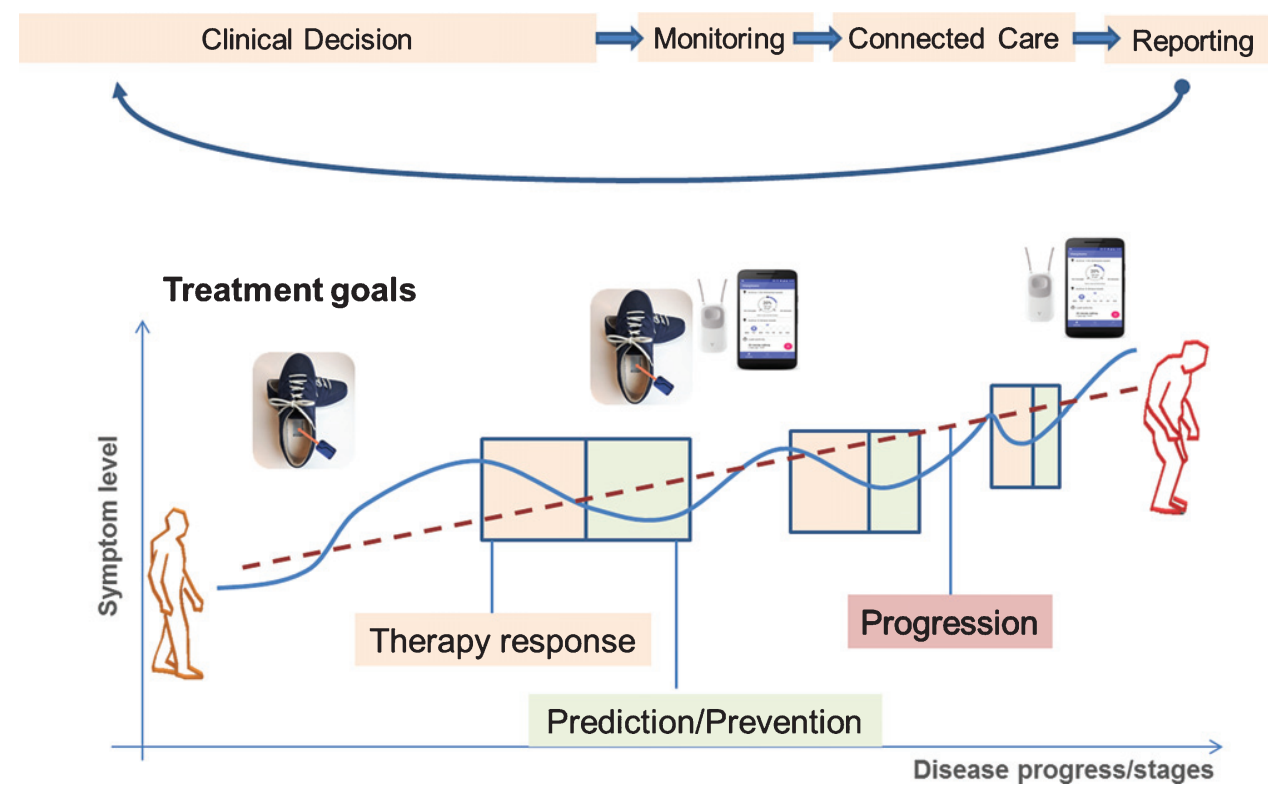

Fig. 1. Digital Health Pathway for Gait and Falls in PD. The patient here is monitored using a combination of wearable sensors equipped with gyroscopes and accelerometers that are incorporated into the subject's own shoes, or into a falls detector (worn as a necklace around the neck) and a smartphone. Two typical real-life care scenarios are illustrated here. Therapy response: the current state of the individual patient indicates a worsening at the symptom levels that necessitates an adaptation of the therapy (e.g., increase or reduce pharmacological treatment, initiate or intensify physiotherapy, etc.). Prediction/prevention: the symptom level is in an optimal state, and there is no need to adjust the therapy, but now the goal is to predict new worsening or development of foreseeable symptoms along natural disease progression (e.g., development of postural instability, increased risk of falling, etc.). Additionally, the overall disease trajectory expressing the theoretical progression rate of the individual patient also taking into account intercurrent events (infections, co-morbidities, operations, etc.) can be deducted from longitudinal and individualized target parameter assessment over the disease course. 
Table 2

Digital Health Pathway workflow and categories

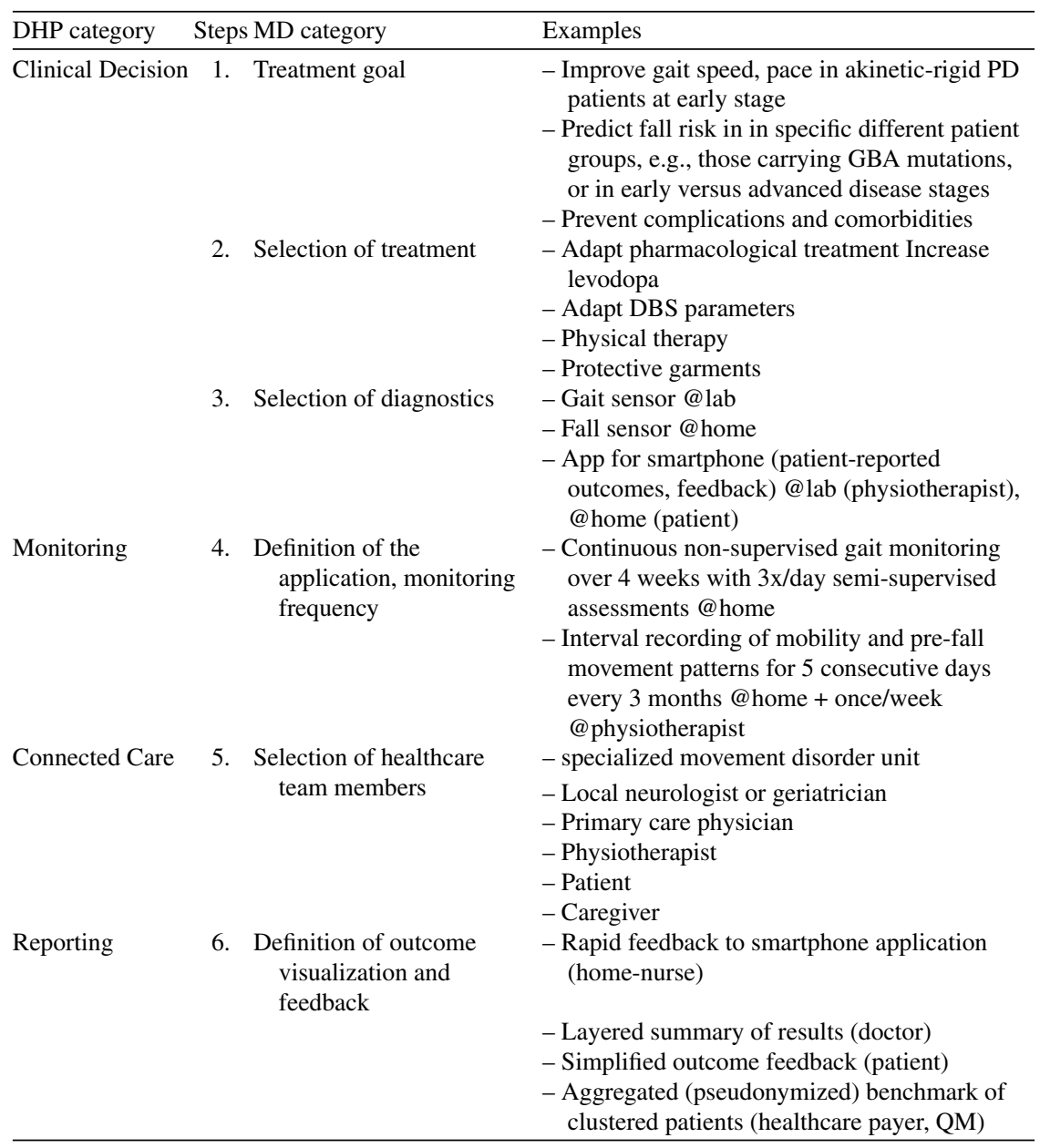

healthcare workflow (Table 2). At each step, relevant data and information have to be selected and fed into the DHP.

The DHP starts with defining the treatment goal which is the target of the clinical decision that should be reached as a joint decision between patient and healthcare provider. Using the example of gait and falls management, this could be to improve gait/mobility, to minimize the risk of falling or reduce the number of falls (Table 2). If the treatment goal is defined, the actual treatment and the related diagnostic healthcare technologies have to be selected to adequately monitor the treatment effects and shape the prediction/prevention state.

At this stage ("clinical decision", Table 2), the relevant medical data or information has to be defined (examples of categories are listed in Table 3): which parameter(s) reports the treatment effect (e.g., target parameter - in RCTs know as primary outcome or endpoint); which parameter(s) define the specific characteristics of the selected patient which is relevant for a selected treatment goal (e.g., stratifier - in RCTs know as inclusion/exclusion criteria, in big-data research known as confounders, in personalized medicine approaches enabling "tailored medicine"); which context information is relevant for the specific characteristics of the measurement and acquisition paradigm (e.g., supervised vs. unsupervised or standardized vs. non-standardized tests, supervised/non-supervised - in RCTs highly standardized by the study protocol); which treatment relevant information from the patient and the doctor are required describing the actual state of treatment (e.g., which treatment for was selected, how does the patient and/or the doctor/therapist rate the current treatment efficiency; in RCTs defined by the test paradigm). 
Table 3

Types of medical data: The medical data (parameter/information) of each category depend on the selected treatment paradigm (TP) and are subject to a constant improvement by classical and digital research

\begin{tabular}{ll}
\hline Parameter/Medical data categories & Example \\
\hline Target parameter & Gait speed, gait regularity (TP=>improve gait) \\
Stratifier & Number of falls (TP=>reduce falls) \\
& tremor-dominant PD, akinetic-rigid PD, presence \\
& of axial symptoms, cognitive function \\
Genotype (e.g., GBA mutation carrier) atypical & parkinsonism (e.g., PSP) \\
& Home assessment, data from the environment \\
& Structured assessment (parcours) \\
Context information & Daytime of assessment \\
& Activities of daily living (during the assessment) \\
& Motor fluctuations, falls \\
& Pharmacological (e.g., increase of dopamine \\
& agonist) \\
Treatment information & Deep brain stimulation (e.g., adaptation of \\
& parameters) \\
& Physiotherapy \\
& Best-ON, Medical-OFF, limiting dyskinesia
\end{tabular}

In the next stage, the individual monitoring paradigm has to be defined. This includes the frequency of assessments, e.g., continuous, semicontinuous, daily, weekly, monthly, which depends on the treatment goal. It also defines the place of testing, e.g., at the doctor's office, at the physiotherapist's practice or in the patient's own home environment.

A very important aspect of the DHP workflow is the selection of the connected care team, including the patient, that needs to be involved in the clinical treatment and outcome assessment for the selected treatment goal. Here, it is important to acknowledge the information needs, in particular the requirements of the patient to be part of the healthcare process and the therapy decision.

For the reporting of the target parameters that reflect the treatment effect, it is necessary to assign relevant context information and then to select who should receive this information, specifying which members of the healthcare team, the patient, or both. At the same time, the type of visualization needs to be defined accordingly, as the way how to report the target parameter to the doctor, allied healthcare professional or patient may vary. It is important to acknowledge that the interest in the parameters and related treatment information especially differs substantially between patients and healthcare providers, which has to be carefully addressed by the data visualization paradigms. One focus of future research has to address the individualization strategy and statistics that provide a personalized reporting in order to support the individual clinical decision.

\section{FUTURE OPTIONS FOR IMPROVED STRATIFICATION}

Sources for defining subgroups of patients not only refer to individualized data capture, but may largely profit from integration of available data sources (e.g., electronic health records) or data from deep phenotyped patient cohorts [22, 23]. Clinicogenetic correlation provided the first entry points for successful stratification in PD. Mutations in the glucocerebrosidase A1 gene (GBA) represent the most relevant genetic stratifier in terms of frequency (more than 5\% of sporadic PD cases) and seem to exhibit a distinct clinical presentation with more rapid disease progression and more axial clinical symptoms [24]. This specifically relates to the aforementioned example of gait impairments (freezing) and falls, that are typically related to cognitive decline in heterozygous carriers of the GBA mutations.

In terms of pharmacogenomics, a recent pilot study identified genetic factors related to a more positive therapeutic outcome in PD patients treated with DBS [25]. Here, a specific alpha-synuclein polymorphism predicted a more positive outcome and is currently investigated in a larger cohort of well-characterized patients treated with DBS [26]. These studies on clinicogenetic stratifiers for pharmacological or neuromodulation therapy still require further validation and indicate that future clinical trials need to focus more on individual rather than averaged therapeutic responses. 


\section{CONCLUSION AND VISION FOR THE NEXT YEARS}

Digital medicine [9] enables new personalized healthcare approaches that require a profound change in medical workflow guidelines. Wearable healthcare technologies including body-worn sensors and smartphone applications can provide better and objective outcome parameters. Their patient-centered application moves the role of the patient even more into the center of healthcare. Individualized feedback for doctors, therapists and the patients themselves will increase transparency and promote the process of shared decision making. As one aspect of digital medicine, we propose the concept of DHPs which will guide and improve the application of wearable healthcare technologies.

One long-term benefit of DHPS will be the standardization of real-life medical data derived from individual patients into longitudinal multimodal disease trajectories. This will allow matching and clustering of different patient trajectories for individualized prediction about the expected treatment efficacy of particular interventions. It will also enable individualized benchmarking for the patient (to rate and understand the individual disease status on his or her journey along the disease course), for the healthcare provider (to control for quality and efficacy of the chosen treatment paradigm), and for society (to secure best medical care for its citizens and to control for efficiency of invested). Ultimately, the growing amount of medical data derived from individualized real-life treatment contexts will enable academic and industrial research to further improve objective outcomes and better tailor individual care.

\section{ACKNOWLEDGMENTS}

The manuscript was supported by an EITHealth grant "MoveIT". JK was supported by a research grant from the Bavarian Ministry of Economics (FallRiskPD), EIT-Health (MoveIT), EIT-Digital (Vital@Home), and by a Fraunhofer Attract grant (DHPs). RK was supported research grants from Fonds National de Recherche de Luxembourg (FNR; PEARL [FNR/P13/6682797] and NCER-PD [FNR/ NCER13/BM/11264123]), and the European Union's Horizon2020 research and innovation program (WIDESPREAD; CENTRE-PD; grant agreement no. 692320; CENTRE-PD). Prof. Bastiaan
R. Bloem was supported by a center grant of the Parkinson's Foundation.

\section{CONFLICT OF INTEREST}

JK has received institutional research grants from Bavarian Research Foundation; Emerging Field Initiative, FAU; EIT-Health; EIT-Digital; EU (H2020), German Research Foundation (DFG); BMBF. Industry sponsored institutional IITs and grants from Teva GmbH; Licher MT GmbH; Astrum IT GmbH; AlphaTelemed AG. He works on advisory boards in the field of healthcare technologies and digital health of different associations of medical professionals, industries, and political authorities. He holds shares of Portabiles HealthCare Technologies GmbH, Portabiles GmbH, Alpha-Telemed AG, and received compensation and honoraria from serving on scientific advisory boards for LicherMT GmbH, Abbvie GmbH, UCB Pharma $\mathrm{GmbH}$, Athenion $\mathrm{GmbH}$, and Thomashilfen $\mathrm{GmbH}$; as well as lecturing from UCB Pharma GmbH, TEVA Pharma GmbH, Licher MT GmbH, Desitin GmbH, Abbvie GmbH, Solvay Pharmaceuticals, and Ever Neuro Pharma GmbH; Dr. Klucken has a patent related to gait assessments pending.

PS's work was supported by the Parkinson's Foundation and has received institutional support from the Patient-Centered Outcomes Research Institute, Robert Wood Johnson Foundation, CHDI, and the Cystic Fibrosis Foundation. He serves on the corporate boards of the Huntington's Disease Study Group, Davis Phinney Foundation, and Night Nurse Triage.

RK serves as Editorial Board Member of the European Journal of Clinical Investigation, the Journal of Parkinsonism and Related Disorders and the Journal of Neural Transmission. Dr. Krüger has received research grants from Fonds National de Recherche de Luxembourg (FNR; PEARL [FNR/P13/6682797/Krüger], MiRisk [C17/BM/11676395], MotaSYN [12719684], MAMaSyn-PD and NCER-PD), the German Research Council (DFG; KR2119/8-1), the Michael J Fox Foundation, the European Union's Joint Program-Neurodegenerative Diseases (JPND; COURAGE-PD), the European Union's Horizon2020 research and innovation program (WIDESPREAD; CENTRE-PD; grant agreement no. 692320) and the the Federal Ministry for Education and Research (BMBF; Mito-PD 031 A $430 \mathrm{~A}$ ), as well as speaker's honoraria and/or travel grants from Abbvie, Zambon and Medtronic. Dr 
Krüger participated as PI or site-PI for industry sponsored clinical trials without receiving honoraria.

BB currently serves as Associate Editor for the Journal of Parkinson's disease, serves on the editorial of Practical Neurology, has received honoraria from serving on the scientific advisory board for Abbvie, Biogen, UCB and Walk with Path, has received fees for speaking at conferences from AbbVie, Zambon and Bial, and has received research support from the Netherlands Organization for Scientific Research, the Michael J Fox Foundation, UCB, Abbvie, the Stichting Parkinson Fonds, the Hersenstichting Nederland, the Parkinson's Foundation, Verily Life Sciences, Horizon 2020, the Topsector Life Sciences and Health, and the Parkinson Vereniging.

\section{REFERENCES}

[1] Tricoci P, Allen JM, Kramer JM, Califf RM, Smith SC Jr (2009) Scientific evidence underlying the ACC/AHA clinical practice guidelines. JAMA 301, 831-841.

[2] Dorsey ER, Vlaanderen FP, Engelen LJ, Kieburtz K, Zhu W, Biglan KM, Faber MJ, Bloem BR (2016) Moving Parkinson care to the home. Mov Disord 31, 1258-1262.

[3] NICE guideline, National Institute for Health and Care Excellence, http://www.nice.org.uk/guidance/ng71,

[4] National Collaborating Centre for Chronic Conditions (UK) (2006) Parkinson's Disease: National Clinical Guideline for Diagnosis and Management in Primary and Secondary Care, Royal College of Physicians (UK), London.

[5] S3 Guidelines for Parkinson's Disease German Society of Neurology, https://www.awmf.org/leitlinien/detail/11/030010.html

[6] Keus SH, Munneke M, Graziano M, Paltaama J, Pelosin E, Domingos J, Bruhlmann S, Ramaswamy B, Prins J, Struiksma C, Rochester L, Nieuwboer A, Bloem BR, On behalf of the Guideline Development Group (2014) European Physiotherapy Guideline for Parkinson's Disease, KNGF / ParkinsonNet, Nijmegen.

[7] Patel T, Chang F, Parkinson Society Canada (2014) Parkinson's disease guidelines for pharmacists. Can Pharm J(Ott) 147, 161-170.

[8] Guidelines on the treatment of non-motor symptoms of Parkinson disease, American Academy of Neurology, https: //www.aan.com/Guidelines/home/ByTopic?topicId=17

[9] Steinhubl RS, Topol E (2018) Digital medicine, on its way to being just plain medicine. NPJ Digit Med 1, Article 20175.

[10] Pasluosta CF, Gassner H, Winkler J, Klucken J, Eskofier BM (2015) An emerging era in the management of Parkinson's disease: Wearable technologies and the internet of things. IEEE J Biomed Health Inform 19, 1873-1881.

[11] Klucken J, Friedl KE, Eskofier B, Hausdorff JM (2015) Enabling technologies for Parkinson's disease management. IEEE J Biomed Health Inform 19, 1775-1776.

[12] Espay AJ, Bonato P, Nahab FB, Maetzler W, Dean JM, Klucken J, Eskofier BM, Merola A, Horak F, Lang AE, Reilmann R, Giuffrida J, Nieuwboer A, Horne M, Little MA, Litvan I, Simuni T, Dorsey ER, Burack MA, Kubota K, Kamondi A, Godinho C, Daneault JF, Mitsi G, Krinke L, Hausdorff JM, Bloem BR, Papapetropoulos S,
Movement Disorders Society Task Force on Technology (2016) Technology in Parkinson's disease: Challenges and opportunities. Mov Disord 31, 1272-1282.

[13] Stamford JA, Schmidt PN, Friedl KE (2015) What engineering technology could do for quality of life in Parkinson's disease: A review of current needs and opportunities. IEEE J Biomed Health Inform 19, 1862-1872.

[14] Hansen C, Sanchez-Ferro A, Maetzler W (2018) Monitoring Parkinson's disease: Technology promises a shift from the clinic to the home. J Parkinsons Dis 8(Suppl 1), S41-S45.

[15] Hunkeler EM, Hargreaves WA, Fireman B, Terdiman J, Meresman JF, Porterfield Y, Lee J, Dea R, Simon GE, Bauer MS, Unutzer J, Taylor CB (2012) A web-delivered care management and patient self-management program for recurrent depression: A randomized trial. Psychiatr Serv 63, 1063-1071.

[16] Campbell H, Hotchkiss R, Bradshaw N, Porteous M (1998) Integrated care pathways. BMJ 316, 133-137.

[17] Rotter T, Kinsman L, James E, Machotta A, Willis J, Snow P, Kugler J (2012) The effects of clinical pathways on professional practice, patient outcomes, length of stay, and hospital costs: Cochrane systematic review and meta-analysis. Eval Health Prof 35, 3-27.

[18] Schlachetzki JCM, Barth J, Marxreiter F, Gossler J, Kohl Z, Reinfelder S, Gassner H, Aminian K, Eskofier BM, Winkler J, Klucken J (2017) Wearable sensors objectively measure gait parameters in Parkinson's disease. PLoS One 12, e0183989.

[19] Marxreiter F, Gassner H, Borozdina O, Barth J, Kohl Z, Schlachetzki JCM, Thun-Hohenstein C, Volc D, Eskofier BM, Winkler J, Klucken J (2018) Sensor-based gait analysis of individualized improvement during apomorphine titration in Parkinson's disease. J Neurol 265, 2656-2665.

[20] Silva de Lima AL, Hahn T, de Vries NM, Cohen E, Bataille L, Little MA, Baldus H, Bloem BR, Faber MJ (2016) Largescale wearable sensor deployment in Parkinson's patients: The Parkinson@ Home Study Protocol. JMIR Res Protoc 5, e172.

[21] EIT-Health project "moveIT", https://www.eithealth.eu/ moveit

[22] Parkinson Progression Marker Initiative (2011) The Parkinson Progression Marker Initiative (PPMI). Prog Neurobiol 95, 629-635.

[23] Hipp G, Vaillant M, Diederich NJ, Roomp K, Satagopam VP, Banda P, Sandt E, Mommaerts K, Mosch S, Longhino L, Schweicher A, Hanff A, Nicolai B, Kolber P, Reiter D, Pavelka L, Binck S, Pauly C, Geffers L, Betsou F, Gantenbein M, Klucken J, Gasser T, Hu M, Balling R, Krueger R (2018) The Luxembourg Parkinson's Study: A comprehensive approach for stratification and early diagnosis. Front Aging Neurosci 10, 326.

[24] Zimmermann M, Gaenslen A, Prahl K, Srulijes K, Hauser AK, Schulte C, Csoti I, Berg D, Brockmann K (2018) Patient's perception: Shorter and more severe prodromal phase in GBA-associated PD. Eur J Neurol, doi: 10.1111/ene. 13776

[25] Weiss D, Herrmann S, Wang L, Schulte C, Brockmann K, Plewnia C, Gasser T, Sharma M, Gharabaghi A, Kruger R (2016) Alpha-synuclein gene variants may predict neurostimulation outcome. Mov Disord 31, 601-603.

[26] Schuepbach WM, Rau J, Knudsen K, Volkmann J, Krack P, Timmermann L, Halbig TD, Hesekamp H, Navarro SM, Meier N, Falk D, Mehdorn M, Paschen S, Maarouf M, Barbe MT, Fink GR, Kupsch A, Gruber D, Schneider 
GH, Seigneuret E, Kistner A, Chaynes P, Ory-Magne F, Brefel Courbon C, Vesper J, Schnitzler A, Wojtecki L, Houeto JL, Bataille B, Maltete D, Damier P, Raoul S, SixelDoering F, Hellwig D, Gharabaghi A, Kruger R, Pinsker MO, Amtage F, Regis JM, Witjas T, Thobois S, Mertens
P, Kloss M, Hartmann A, Oertel WH, Post B, Speelman $\mathrm{H}$, Agid Y, Schade-Brittinger C, Deuschl G, EARLYSTIM Study Group (2013) Neurostimulation for Parkinson's disease with early motor complications. $N$ Engl J Med 368, 610-622. 